\title{
The Influence of Vaccination of Broiler Chickens and Turkeys with Live E. coli Attenuated Vaccine on E. coli Population Properties and TRT Vaccination Efficacy
}

\author{
Marcin Śmiałek*(D), Joanna Kowalczyk (1) and Andrzej Koncicki \\ Department of Poultry Diseases, Faculty of Veterinary Medicine, University of Warmia and Mazury, \\ Oczapowskiego 13, 10-719 Olsztyn, Poland; welencasia@gmail.com (J.K.); koncicki@uwm.edu.pl (A.K.) \\ * Correspondence: marcin.smialek@uwm.edu.pl; Tel.: +48-089-523-38-11
}

Citation: Śmiałek, M.; Kowalczyk, J.; Koncicki, A. The Influence of Vaccination of Broiler Chickens and Turkeys with Live E. coli Attenuated Vaccine on E. coli Population Properties and TRT Vaccination Efficacy. Animals 2021, 11, 2068. https://doi.org/10.3390/ani11072068

Academic Editor: Andrew Peters

Received: 10 May 2021

Accepted: 6 July 2021

Published: 11 July 2021

Publisher's Note: MDPI stays neutral with regard to jurisdictional claims in published maps and institutional affiliations.

Copyright: (c) 2021 by the authors. Licensee MDPI, Basel, Switzerland. This article is an open access article distributed under the terms and conditions of the Creative Commons Attribution (CC BY) license (https:/ / creativecommons.org/licenses/by/ $4.0 /)$.
Simple Summary: Escherichia coli infections are considered one of the major causes of economic loss in the poultry industry. The reasons for the magnitude of the problem are the numerous sources of infection with these bacteria for birds and the need for an effective prevention method. Vaccination is one of the strategies for minimizing the consequences of $E$. coli infection. In this study, we performed three independent experiments at farm level using a live vaccine against $E$. coli. Antibiotic-free broiler chickens, conventional broiler chickens and broiler turkeys were examined in different experiments. The most meaningful results and conclusions of these experiments are that vaccination against colibacillosis decreases the population count of E. coli, increases the antibiotic susceptibility of field $E$. coli isolates and has no impact on the efficacy of vaccination against another significant poultry upper respiratory tract disease-TRT. We believe that the vaccination of broiler chickens and turkeys against E. coli can improve bird health and should be considered in terms of routine immunoprophylaxis.

Abstract: Colibacillosis is one of the major causes of economic losses in the poultry industry. Vaccination against $E$. coli is attracting increasing interest. The aim of the study was to evaluate the influence of vaccination with live, aro $A$ gene-deleted vaccine on the structure and properties of field $E$. coli population and its potential impact on TRT vaccination efficacy in broiler chickens and turkeys. We performed three independent experiments on farms: (1) with antibiotic-free broiler chickens, (2) with conventional broiler chickens and (3) with broiler turkeys. In experiment 1, we have recorded an approx. $0-15 \%$ prevalence of multi-susceptible $E$. coli strains in the first production cycle. Starting from production cycle number two, after vaccination introduction, successive significant increases in E. coli susceptibility emerged, reaching $100 \%$ of strains at the end of production cycle 3 . Increased $E$. coli susceptibility remained for three production cycles after vaccination withdrawal. In experiments 2 ( 2 production cycles) and 3 ( 1 production cycle), we recorded similar tendencies of E. coli susceptibility profile change. In experiments 1 and 2, the E. coli population count was lower after vaccination. In experiments 2 and 3, no negative influence of $E$. coli vaccination on the level of specific antibodies against TRT was recorded.

Keywords: broiler chickens; broiler turkeys; E. coli vaccination; E. coli antibiotic susceptibility; TRT vaccination

\section{Introduction}

Avian pathogenic Echerichia coli (APEC) is a causative agent of poultry colibacillosis. The disease has been one of the major causes of economic losses in the poultry industry worldwide. These losses are due to mortality and decreased productivity of affected birds [1]. Escherichia coli infections are usually secondary disruptors of homeostasis where that is primarily triggered by other diseases, immunosuppression or disorders in respiratory system functioning [2]. The E. coli serotypes which are considered to be particularly pathogenic are $\mathrm{O} 1, \mathrm{O} 2, \mathrm{O} 78, \mathrm{O} 8$, and $\mathrm{O} 35$ and it is these which are most often isolated from 
infected birds. The transmission of infection is usually horizontal, with the main source being the contaminated housing environment [2].

The prevention of colibacillosis is problematic, especially given that no effective method has been developed so far. Colibacillosis prevention is largely a matter of good sanitation, hygiene, and elimination of the sources of infection [1], as well as negation of factors that increase the risk of its development in a flock [3].

Recently, an effective commercial vaccine based on the O78:K80 E. coli strain with aro $A$ gene deletion has been developed and used in the field. Its efficacy has been proven in several studies, particularly in chickens but also in turkeys. The vaccine has been demonstrated to decrease the risk of mortality and development of colibacillosis-related lesions in chickens and turkeys after APEC experimental infection when administered on the first or fifth day of life $[4,5]$. Although the highest protection was observed against the homologous infection (O:78), to some extent the vaccine also induced cross-protection against other E. coli serotypes [4]. Our previous study [6] demonstrated that the vaccination of broiler chickens with this vaccine increased their production profitability. Vaccination against $E$. coli has been shown not to interfere with the effectiveness of vaccinations against other poultry diseases, such as IB (infectious bronchitis), IBD (infectious bursal disease), or ND (Newcastle disease) [6,7].

Another issue associated with poultry APEC infections is the multi-drug resistant (MDR) and extended-spectrum beta-lactamase (ESBL)-producing E. coli strains, which are increasingly commonly isolated from poultry colibacillosis cases [8-10]. These strains can pose a direct risk to consumers since they can be passed to humans via the food chain or by direct contact, and they can also pass antibiotic resistance genes to other bacteria [11].

Our previous study [6] demonstrated that spray vaccination of day-old broilers against E. coli in the sixth week of life decreased the number of isolates of the bacteria from internal organs excluding those of the respiratory system and that the E. coli strains isolated from the vaccinated birds were more susceptible to antimicrobials. These results indicate that vaccination can decrease the risk and level of infection and that improve antibiotic susceptibility of field E. coli strains.

Considering the need for prevention of colibacillosis and the supposed inimicality of E. coli vaccination to MDR, a study was undertaken under field conditions to verify the results of previous studies addressing the influence of $E$. coli vaccination on a decreased field E. coli population count and the improvement of their antimicrobial susceptibility. The study was carried out with three experimental models, which were antibiotic-free broiler chickens, conventional broiler chickens, and broiler turkeys. It additionally evaluated the influence of $E$. coli vaccination on the efficacy of simultaneous vaccination against avian metapneumoviruses (aMPV) in chickens and turkeys.

\section{Materials and Methods}

\subsection{Ethic Statement}

According to information from the Local Ethics Committee in Olsztyn, no special approval was necessary for the experiments performed under field conditions. All animal procedures, vaccination, and sample collection were performed during standard veterinary inspections and observations.

\subsection{Experimental Layout}

\subsubsection{Experiment 1}

Experiment 1 was carried out on farm 1, operating only one chicken house $(\mathrm{CH} 1)$ with the capacity of 35,000 broiler chickens. It was an antibiotic-free farm, on which no therapeutic antibiotics had been used for at least two years prior to the experiment. Hence, experiment 1 entailed no use of antibiotics. It was staged over six subsequent production cycles. In the first three (production cycles 1-3), all birds on were vaccinated against colibacillosis. The vaccination was performed on the farm on the first day of life immediately after they had arrived at $\mathrm{CH} 1$ in transport boxes. The chicks were vaccinated 
against $E$. coli with a coarse spray at a dose recommended by the producer and observed for $15 \mathrm{~min}$. Afterwards, they were allowed out of the boxes onto litter. Vaccination against E. coli was not continued in the last three production cycles (production cycles 4-6). The chicks were also vaccinated at the hatchery against IB using a mixture of the Mass-like and 793B strains with a coarse spray throughout the entire experiment. Over the six production cycles, swabs were taken for microbiological analyses from the internal organs of 3 randomly selected birds in the third and sixth weeks of life. At each sampling, swabs from three birds were preserved for analyses. The samples were collected immediately after diagnostic euthanasia of the birds under sterile conditions using dry swabs without transport media. Peritoneum, liver, lungs, heart, jejunum, hip and hock joint, trachea, and suborbital sinus swabs were taken. Individual swabs were transferred for microbiological analyses with semiquantitative determination of the E. coli colony count, and the isolated E. coli strains were evaluated for their susceptibility to a panel of 20 antimicrobials.

\subsubsection{Experiment 2}

Experiment 2 was carried out on farm 2 on which two chicken houses were in use (CH2 and $\mathrm{CH} 3$ ) with capacity of approximately 25,000 birds each. The birds from $\mathrm{CH}_{2}$ were vaccinated against $E$. coli on the 10th day of life with a coarse spray, at a dose recommended by the producer. Birds from $\mathrm{CH}_{3}$ served as the control. The birds from both chicken houses were vaccinated against IB at the hatchery with the Mass-like and 793B strain mixture (common vaccination schedule against IB), and also against TRT using Clone $\mathrm{K}$ of aMPV subtype A, at a dose recommended by the producer. The house 2 birds were vaccinated against $E$. coli and TRT with one preparation made by mixing both vaccines in one diluent. Experiment 2 was continued for two subsequent production cycles. In weeks 3 and 6 of life in the first production cycle and in week 6 of life in the second production cycle, swabs were collected for microbiological analyses from the internal organs of three randomly selected birds each from $\mathrm{CH} 2$ and $\mathrm{CH} 3$. The swab types and sampling method were the same as those described in experiment 1 . During both production cycles, 23 blood samples were additionally taken from birds kept in $\mathrm{CH} 2$ and $\mathrm{CH} 3$ in week 6 of life for serological analyses aimed at determining the level of specific antibodies against aMPV.

\subsubsection{Experiment 3}

Experiment 3 was carried out on a two-turkey-house farm of male broiler turkeys with approximately 3500 birds in each house. This experiment was performed during one production cycle. The turkeys from turkey house 1 (TH1) were vaccinated against E. coli twice, i.e., on the first day and in the third week of life. The vaccination procedure was the same as in experiments 1 and 2. All of the birds were also vaccinated against TRT using Clone $\mathrm{K}$ of aMPV subtype A at a dose recommended by the producer. In TH1, the vaccination against $E$. coli and TRT on the first day and in the third week of life was administered in a mixture of both vaccines in one diluent. In week 3 and 6 of life, samples were collected for microbiological analyses from the birds' internal organs as in experiments 1 and 2 . In addition, in weeks 10 and 14 of life, swabs were collected from the nasal cavity, trachea, and cloaca of six live birds from both turkey houses for microbiological analyses. In weeks 6 and 14 of life, 23 blood samples were additionally taken from birds kept in both turkey houses for serological analyses aimed at determining the level of specific antibodies against aMPV.

\subsection{Antimicrobial Use on Experimental Farms}

In each experiment, antimicrobials were not administrated to birds for at least 10 days after $E$. coli vaccination. In experiment 1, no antimicrobials were used throughout the entire study. In experiments 2 and 3, the antimicrobial treatment scheme was the same for both houses in individual experiments $(\mathrm{CH} 2$ and $\mathrm{CH} 3$ in experiment 2 and TH1 and TH2 in experiment 3$)$. 


\subsection{Birds and Vaccination}

In experiments 1 and 2, chicken houses were populated with Ross 308 broiler chicks of both sexes purchased from one hatchery (which changed between experiment 1 and experiment 2) one hatch, and one reproduction flock.

In experiment 3, turkey houses were settled with male B.U.T. 6 turkey poults, purchased from one hatchery and from one hatch. If the source of the eggs was one reproduction flock or several was unascertainable.

A live, attenuated, aroA gene-deleted E. coli vaccine (Zoetis, Madison, NJ, USA) was used in all experiments. The vaccine comprises the viable E. coli O78 strain. The vaccine strain from the same batch as the vaccines used in all experiments was analyzed for antimicrobial susceptibility with the same method used on the isolated field strains. Feed and water were given to the birds ad libitum in both experiments.

\subsection{Microbiological Studies}

Microbiological studies were performed as described previously [6]. Briefly, after $24 \mathrm{~h}$ of pre-incubation in tryptic soy broth (Argenta, Poznań, Poland) at $40.5^{\circ} \mathrm{C}$, further incubation proceeded at the same temperature for $24 \mathrm{~h}$ on Columbia agar with $5 \%$ addition of defibrinated sheep blood and MacConkey agar media (Argenta, Poznań, Poland). After morphological and biochemical evaluation, E. coli isolates were evaluated for their susceptibility to a panel of 20 antimicrobials, according to the Clinical and Laboratory Standards Institute guidelines [12]. The following antimicrobials were analyzed: amoxycillin (amx), amoxycillin + clavulanic acid (amc), ceftiofur (cef), clindamycin (cli), colistin sulfate (cst), doxycycline (doxy), enrofloxacin (enr), erythromycin (ery), gentamycin (gen), florfenicol (ff), flumequine (fluq), lincomycin/spectinomycin (ls), marbofloxacin (mbf), neomycin (nm), norfloxacin (nor), oxytetracycline (otc), penicillin $G$ (pen), sulfamethoxazole/trimethoprim (sxt), tetracycline (tet), and tylosin (tyl) (Argenta, Poznań, Poland). Susceptibility was assessed on a scale of $0-3$, where 0 denoted no susceptibility. The strains that were sensitive to $35 \%$ ( 7 or more) of the antimicrobials tested with a susceptibility level of at least 1 were referred to as multisensitive strains, as described previously [6].

The results of the microbiological analyses are presented as the mean number of $E$. coli isolates from internal organs or swabs of vaccinated or control birds at different periods of the experiment. The results of antimicrobial susceptibility testing are presented as the mean percentage of multisensitive strains among all $E$. coli isolated strains at different periods of the experiment. Additionally, panel testing results are presented as the mean number of antimicrobials the isolated $E$. coli were susceptible to.

\subsection{Serological Evaluation}

A commercial ELISA APV Ab Test kit (IDEXX Laboratories, Westbrook, MN, USA) was used to determine the titer of TRT-specific IgY in broiler and turkey serum in experiments 2 and 3 , respectively. Individual stages of the tests were performed with an epMotion $5075 \mathrm{LH}$ liquid handler (Eppendorf, Hamburg, Germany), an EL $\times 405$ automatic multi-well plate washer (BioTek Instruments, Winooski, VT, USA), and an EL $\times 800$ plate reader (BioTek Instruments). The mean geometric titer of antibodies and $\mathrm{CV} \%$ were calculated for each group in each sampling period.

\subsection{Statistical Analysis}

Statistical analysis was performed with GraphPad Prism 6.05 (GraphPad Software, San Diego, CA, USA) using the Mann-Whitney $U$ test. Differences were considered statistically significant at $p<0.05$.

\section{Results}

\subsection{Experiment 1}

During the first production cycle in the experiment 1, the mean number of E. coli isolates reached 8.67 and 9.00 in the 3rd and 6th weeks of life, respectively. The number 
of $E$. coli isolates decreased during production cycle 2 but the differences between isolate totals in the first and second production cycles for respective sampling periods were not statistically significant. Starting from production cycle 3, the mean number of E. coli isolates from the internal organs of birds decreased significantly and remained significantly lower during production cycles 4-6 in comparison to the first cycle at both sampling periods (week 3 and 6). The results of $E$. coli isolation from experiment 1 bird samples are summarized in Table 1.

Table 1. Summarized results of E. coli isolation from experiment 1 bird samples.

\begin{tabular}{cccc}
\hline \multirow{2}{*}{ Production Cycle Number } & Bird Age-Week of Life & $\begin{array}{c}\text { Mean Number of } \\
\text { E. coli Isolates } \pm \text { SD }\end{array}$ & $p$ Value \\
\hline 1 & 3 & $8.67 \pm 0.58$ & - \\
\cline { 2 - 4 } & 6 & $9.0 \pm 0.0$ & - \\
\hline \multirow{2}{*}{2} & 3 & $7 \pm 1.73$ & 0.299 \\
\hline \multirow{2}{*}{3} & 6 & $3.0 \pm 1.0$ & 0.091 \\
\hline \multirow{2}{*}{4} & 3 & $2.0 \pm 0.0 *$ & 0.025 \\
\hline \multirow{2}{*}{5} & 6 & $3 \pm 2.65$ & 0.059 \\
\hline & 3 & $3.67 \pm 1.53 *$ & 0.037 \\
\hline \multirow{2}{*}{6} & 6 & $3.0 \pm 1.73 *$ & 0.027 \\
\hline & 3 & $3.33 \pm 1.53^{*}$ & 0.015 \\
\hline & 6 & $2.33 \pm 0.58^{*}$ & 0.025 \\
\hline & 3 & $2.0 \pm 0.0 *$ & 0.015 \\
\hline
\end{tabular}

* Values significantly different in comparison to results from production cycle 1 in the same sampling period Results were considered statistically significantly different if $p<0.5$.

The results of antimicrobial susceptibility testing of the isolated E. coli strains are summarized in Table 2. During the first production cycle, the mean number of antimicrobials to which the E. coli isolates displayed susceptibility reached 5.00 and 2.27 in weeks 3 and 6 of life, respectively. In production cycles $2-3$, in each sampling period the mean number of antimicrobials the E. coli strains were susceptible to increased significantly (except for production cycle 3 birds in the third week of life) and peaked at the end of this stage reaching 11.0 antimicrobials. In production cycles $4-6$, the mean susceptibility decreased slightly in comparison to production cycle 3, but still remained significantly higher than the mean in the first production cycle. At the same time, the percentage of multisusceptible isolates was elevated from production cycle 2 and remained so throughout the entire experiment (Table 2).

\subsection{Experiment 2}

Throughout experiment 2, the mean number of E. coli isolates was lower in the vaccinated birds, while the percentage of multisusceptible strains was elevated compared to the percentage of such in the control broilers. In each sampling period of both production cycles, the mean number of antimicrobials the E. coli isolates were susceptible to was significantly higher in the vaccinated than in the control birds. The results of microbiological studies from experiment 2 are summarized in Table 3.

The results of serological evaluation of the mean antibody titers against aMPV from experiment 2 are summarized in Table 4 . In both production cycles, no differences in the mean anti-aMPV antibody titers were recorded between $E$. coli-vaccinated chickens and control birds. 
Table 2. Summarized results of E. coli antimicrobial susceptibility testing from experiment 1 .

\begin{tabular}{|c|c|c|c|c|}
\hline $\begin{array}{c}\text { Production Cycle } \\
\text { Number }\end{array}$ & $\begin{array}{l}\text { Bird Age-Week } \\
\text { of Life }\end{array}$ & $\begin{array}{c}\text { Percentage of } \\
\text { Multisusceptible } \\
\text { Strains }^{1}\end{array}$ & $\begin{array}{l}\text { Mean Number of } \\
\text { Antimicrobials to Which } \\
\text { E. coli Strains Displayed } \\
\text { Susceptibility } \pm \text { SD }\end{array}$ & $p$ Value $^{2}$ \\
\hline \multirow{2}{*}{1} & 3 & $15.38 \%$ & $5.0 \pm 1.62$ & - \\
\hline & 6 & $0 \%$ & $2.27 \pm 0.83$ & - \\
\hline \multirow{2}{*}{2} & 3 & $42.86 \%$ & $6.67 \pm 1.15$ & 0.024 * \\
\hline & 6 & $66.67 \%$ & $7.11 \pm 1.17$ & $<0.0001^{*}$ \\
\hline \multirow{2}{*}{3} & 3 & $33.33 \%$ & $6.33 \pm 0.52$ & 0.111 \\
\hline & 6 & $100 \%$ & $11.0 \pm 1.12$ & $<0.0001$ * \\
\hline \multirow{2}{*}{4} & 3 & $90.91 \%$ & $8.55 \pm 1.81$ & $0.0002 *$ \\
\hline & 6 & $66.67 \%$ & $9.33 \pm 2.65$ & $0.0002 *$ \\
\hline \multirow{2}{*}{5} & 3 & $90 \%$ & $8.3 \pm 1.64$ & $0.0013 *$ \\
\hline & 6 & $42.86 \%$ & $6.29 \pm 2.69$ & $0.0075^{*}$ \\
\hline \multirow{2}{*}{6} & 3 & $100 \%$ & $9.17 \pm 1.47$ & $0.002 *$ \\
\hline & 6 & $42.86 \%$ & $7.57 \pm 1.72$ & $<0.0001 *$ \\
\hline
\end{tabular}

${ }_{1}^{1}$ if a given E. coli strain was susceptible to 7 or more antimicrobials out of 20 tested, the strain was classified as multisusceptible. ${ }^{2} p$ value was calculated based on the mean number of antimicrobials to which the E. coli strains displayed susceptibility. * statistically significant difference between the mean number of antimicrobials to which E. coli displayed susceptibility, between results for birds of particular production cycles (2-6) and ages ( 3 or 6 weeks) and birds from production cycle number 1 and of particular ages ( 3 or 6 weeks). Results were considered statistically significantly different if $p<0.05$.

Table 3. Summarized results of microbiological studies from experiment 2.

\begin{tabular}{|c|c|c|c|c|c|c|}
\hline $\begin{array}{c}\text { Production } \\
\text { Cycle Number }\end{array}$ & $\begin{array}{c}\text { Bird } \\
\text { Age-Week }\end{array}$ & $\begin{array}{l}\text { Chicken } \\
\text { House }\end{array}$ & $\begin{array}{c}\text { Mean E. coli } \text { Isolates } \\
\text { Count } \pm \text { SD }\end{array}$ & $\begin{array}{c}\text { Percentage of } \\
\text { Multi-Susceptible } \\
\text { Strains }^{1}\end{array}$ & $\begin{array}{l}\text { Mean Number of } \\
\text { Antimicrobials to Which } \\
\text { E. coli Strains Displayed } \\
\text { Susceptibility to } \pm \text { SD }\end{array}$ & $p$ Value $^{2}$ \\
\hline \multirow{4}{*}{1} & \multirow{2}{*}{3} & 2-vaccinated & $4.33 \pm 2.31$ & $76.92 \%$ & $8.69 \pm 3.01$ & $<0.0001$ * \\
\hline & & 3-control & $6.33 \pm 1.15$ & $15.79 \%$ & $4.42 \pm 1.68$ & - \\
\hline & \multirow{2}{*}{6} & 2-vaccinated & $3.33 \pm 2.08$ & $90.0 \%$ & $9.30 \pm 2.21$ & $<0.0001$ * \\
\hline & & 3-control & $5.33 \pm 1.53$ & $0 \%$ & $3.06 \pm 0.57$ & - \\
\hline \multirow{2}{*}{2} & \multirow{2}{*}{6} & 2-vaccinated & $2.00 \pm 0.00$ & $50 \%$ & $6.17 \pm 0.98$ & $<0.0001$ * \\
\hline & & 3-control & $2.67 \pm 1.53$ & $0 \%$ & $2.63 \pm 0.52$ & - \\
\hline
\end{tabular}

${ }^{1}$ if a given E. coli strain was susceptible to 7 or more antimicrobials out of 20 tested, the strain was classified as multisusceptible. ${ }^{2} p$ value was calculated based on the mean number of antimicrobials to which the E. coli strains displayed susceptibility. ${ }^{*}$ statistically significant difference between the mean number of antimicrobials to which $E$. coli displayed susceptibility and between vaccinated and control birds in particular stages of the experiment. Results were considered statistically significantly different if $p<0.05$.

Table 4. Results of aMPV serological evaluation in E. coli vaccinated and control unvaccinated birds in experiments 2 and 3.

\begin{tabular}{|c|c|c|c|c|c|}
\hline \multirow{2}{*}{ Experiment } & \multirow{2}{*}{ Production Cycle } & \multirow{2}{*}{ Week } & \multirow{2}{*}{ Parameter } & \multicolumn{2}{|c|}{ Chicken/Turkey House } \\
\hline & & & & Vaccinated & Not Vaccinated \\
\hline \multirow{4}{*}{2} & \multirow{2}{*}{1} & \multirow[b]{2}{*}{6} & Gmean & 242 & 312 \\
\hline & & & $\mathrm{CV} \%$ & 82.8 & 86.2 \\
\hline & \multirow{2}{*}{2} & \multirow{2}{*}{6} & Gmean & 221 & 241 \\
\hline & & & $\mathrm{CV} \%$ & 83.6 & 87.9 \\
\hline \multirow{4}{*}{3} & \multirow{4}{*}{1} & \multirow[b]{2}{*}{6} & Gmean & 276 & 203 \\
\hline & & & $\mathrm{CV} \%$ & 157.4 & 131.1 \\
\hline & & \multirow{2}{*}{14} & Gmean & 391 & 312 \\
\hline & & & $\mathrm{CV} \%$ & 78.2 & 122.2 \\
\hline
\end{tabular}




\subsection{Experiment 3}

The results of microbiological analyses from experiment 3 are summarized in Table 5 . In most of the sampling periods of this experiment, the mean E. coli isolates count in the vaccinated birds was higher than or equal to the count in the control animals. Starting from the sixth week of the turkeys' lives, the percentage of multisusceptible strains increased in the vaccinated birds and peaked in the 14th week of life, reaching $72.22 \%$ of all isolates. In weeks 6 and 14, a significant increase was recorded in the mean number of antimicrobials the E. coli isolates from the E. coli-vaccinated turkeys were susceptible to in comparison to this parameter in control turkeys.

Table 5. Summarized results of microbiological studies from experiment 3.

\begin{tabular}{|c|c|c|c|c|c|c|}
\hline $\begin{array}{l}\text { Production } \\
\text { Cycle Number }\end{array}$ & $\begin{array}{l}\text { Bird Age-Week } \\
\text { of Life }\end{array}$ & Turkey House & $\begin{array}{c}\text { Mean } E \text {. coli } \text { Isolates } \\
\text { Count } \pm \text { SD }\end{array}$ & $\begin{array}{c}\text { Percentage of } \\
\text { Multisusceptible } \\
\text { Strains }^{3}\end{array}$ & $\begin{array}{c}\text { Mean Number of } \\
\text { Antimicrobials to Which } \\
\text { E. coli Strains Displayed } \\
\text { Susceptibility to } \pm \text { SD }\end{array}$ & $p$ Value $^{4}$ \\
\hline \multirow{8}{*}{1} & \multirow{2}{*}{$3^{1}$} & 1 -vaccinated & $6.33 \pm 0.58$ & $0 \%$ & $3.21 \pm 0.98$ & 0.887 \\
\hline & & 2 -control & $5.00 \pm 2.65$ & $13.33 \%$ & $3.13 \pm 2.10$ & - \\
\hline & \multirow{2}{*}{6} & 1-vaccinated & $3.33 \pm 0.58$ & $30 \%$ & $5.50 \pm 1.27$ & $0.027 *$ \\
\hline & & 2 -control & $3.00 \pm 1.00$ & $0 \%$ & $3.22 \pm 1.56$ & - \\
\hline & \multirow{2}{*}{$10^{2}$} & 1 -vaccinated & $2.84 \pm 0.41$ & $11.76 \%$ & $2.18 \pm 2.10$ & 0.084 \\
\hline & & 2 -control & $3.00 \pm 0.00$ & $0 \%$ & $1.28 \pm 0.46$ & - \\
\hline & \multirow{2}{*}{14} & 1-vaccinated & $3.00 \pm 0.00$ & $72.22 \%$ & $7.61 \pm 2.30$ & $<0.0001$ * \\
\hline & & 2 -control & $3.00 \pm 0.00$ & $0 \%$ & $2.06 \pm 1.47$ & - \\
\hline
\end{tabular}

${ }^{1}$ in weeks 3 and 6 swab samples were collected from 9 internal organs of euthanised birds $(n=3) .{ }^{2}$ in weeks 10 and 14 swabs were collected from nasal turbinates, trachea and cloaca from live birds $(n=6) .{ }^{3}$ if a given $E$. coli strain was susceptible to 7 or more antimicrobials out of 20 tested, the strain was classified as multisusceptible. ${ }^{4} p$ value was calculated based on the mean number of antimicrobials to which the E. coli strains displayed susceptibility. ${ }^{*}$ statistically significant difference between the mean number of antimicrobials to which E. coli displayed susceptibility and between vaccinated and control birds in particular stages of the experiment. Results were considered statistically significantly different if $p<0.05$.

The results of serological evaluation of the mean antibody titers against aMPV from experiment 3 are summarized in Table 4 . In every sampling period of experiment 3 , no differences in the mean anti-aMPV antibody titers were recorded between the E. colivaccinated and the control birds.

\section{Discussion}

Colibacillosis has been one of the major causes of economic losses in the poultry production. The key points in controlling avian colibacillosis are management interventions, infection controls, and vaccination strategies $[2,13]$.

The number of cases of infections with multi-drug resistant and ESBL-producing E. coli has recently increased [8-10]. This situation poses a severe threat to consumers because these bacteria can act as donors of antibiotic resistance genes to other bacteria [11].

In the current study no clinical cases of colibacillosis were recorded in none of the experiments and in none of the production cycles. Despite this issue high number of E. coli strains were isolated from experimental birds which allow further evaluation of the properties of those strains. Selection and randomization of birds for laboratory analysis were completed based on the body weight of the birds which had to be equal to the mean body weight of the bird in the chicken house, as well as on the clinical condition of the birds selected for laboratory analyses, which had to be representative of the flock (birds displaying a clinical condition that could be interpreted as a reflection of the general clinical condition of birds in a chicken house were selected).

It has been demonstrated that vaccination against colibacillosis contributed to a reduction in the amount of $E$. coli in the population of vaccinated birds [6,14]. To some extent, our study confirms those observations as we noted a similar tendency in the number of $E$. coli isolates in broiler chickens from experiments 1 and 2 . In experiment 3 , the numbers 
of E. coli isolates were similar in both the vaccinated and the control turkeys. It should be borne in mind, however, that in this experiment, the nasal cavity, trachea, and cloaca swabs were sampled from live birds from week 6 of life. Śmiałek et al. [6] noted fewer isolated E. coli bacteria for most of the internal organs, but not for the respiratory system. It was demonstrated previously that after E. coli vaccination an immune system in the upper respiratory tract is stimulated which in turn results in the local production of specific IgA and stimulation of $\mathrm{CD}^{+} \mathrm{T}$ cells, which was suggested to be the mechanism behind vaccine efficacy in the minimization of $E$. coli spreading to different internal organs from the respiratory tract, after the infection [15]. We cannot exclude that this was also an issue in our study, which would explain those dependencies, however none of the E. coli strains from the vaccinated birds possessed the same antimicrobial susceptibility profile as the vaccine strain does. The above-mentioned data suggest that after vaccination of birds under farm conditions the stimulation of upper respiratory tract immune system is efficient enough to minimize the relocation of the field E. coli from the gate of the infection (respiratory tract) to other internal organs. This would also explain the results which were obtained in experiment 3 with broiler turkeys.

In our previous study, we have demonstrated that $E$. coli vaccination of broiler chickens contributed to a successive increase in antimicrobial susceptibility of isolated E. coli strains [6]. Those findings are consistent with the results of experiments conducted in the present study, including also these obtained for broiler turkeys. The observed phenomenon could be the reason of vaccine and field E. coli strains mutual gene transmission which results in the increased antimicrobial susceptibility of the latter. Considering the restoration of the susceptibility to antimicrobials, interesting data were presented for poultry coccidiosis. The introduction of a population of pathogen with a high antimicrobial susceptibility spectrum into resistant population can, contribute to the increased susceptibility of the pathogen field population [16]. This can be achieved by vaccinating the birds with live vaccines containing Eimeria spp. [17,18].

Interesting conclusions could be drawn from the results of experiment 1 , where those phenomena were investigated in antibiotic-free broiler chickens. As it transpired, even though antibiotics had not been used at this farm for a long time prior to the experiment, highly antibiotic resistant $E$. coli strains were indeed isolated from chickens in the first production cycle. As the vaccination with live $E$. coli vaccine continued, we noted a successive increase in antimicrobial susceptibility of these strains. High susceptibility among E. coli isolates was also determined in samples collected during the three consecutive production cycles after vaccination withdrawal, which undoubtedly points to the modulating effect of the vaccination on the field population of E. coli. In this study we have differentiated the field $E$. coli isolates from the vaccine one, based on the results of antimicrobial susceptibility profiles, as described previously [6]. Similar to the outcome of our previous study [6], the antibiotic susceptibility profiles of E. coli strains isolated from birds from all experiments were different from the susceptibility profile of the vaccine E. coli strain (data not shown; the vaccine strain was susceptible to 15 out of 20 antimicrobials tested). Additionally, the vaccine producer states that the E. coli strain used in the vaccine can be detected in the cloaca swabs of vaccinated birds for no longer than five weeks after the vaccination and that it is not possible to carry over the strain from one production cycle to a subsequent one. The data given above and the results of our studies exclude the possibility of vaccine bacteria re-isolation in microbiological studies in these experiments. Śmiałek et al. [6] also noted a significantly lower amount of antibiotics used in the flocks of birds vaccinated against colibacillosis. In the present study, the schemes of use and doses of antibiotics (used in experiments 2 and 3) were the same for the vaccinated and the control birds. This was expected to exclude the impact of antibiotic therapy on the ultimate results obtained for E. coli population count and properties.

From previous reports, it can be concluded that vaccination against $E$. coli did not affect the immune response to different concurrent viral vaccines such as those against IBD, ND and IB [6,7]. The present study additionally confirmed a lack of such dependencies 
in the case of vaccination against TRT in both broiler chickens and broiler turkeys. The vaccines based on the subtype A of avian metapneumoviruses are known for their low immunogenicity when stimulating the production of specific post-vaccination antibodies detected with the ELISA test $[19,20]$, which explains why the results of the present study show low titers of post-vaccination antibodies in the examined birds. In our experiment, we found no significant differences in the levels of these antibodies between the control and E. coli-vaccinated groups of both chickens and turkeys. Interestingly, considering the often-overlapping scheme of vaccination against TRT and E. coli in field veterinary practice, it is worth emphasizing that the vaccines used in the present study were prepared in one diluent and administered to birds simultaneously via a coarse spray. This experimental scheme was expected to mirror the field practices as much as possible.

\section{Conclusions}

From the presented results of the three independent experiments, it can be concluded that vaccination of poultry against colibacillosis with the use of a live, gene-deleted vaccine improves the antimicrobial susceptibility of field E. coli isolates, and also it lowers the E. coli population count. Vaccination against the bacteria had no negative impact on the efficacy of simultaneous TRT vaccination. Very similar results were recorded with respect to both broiler chickens and turkeys. The mechanisms of modulating the impact of the vaccine E. coli strain on the antimicrobial susceptibility of field bacteria population remain unelucidated and definitely merit further studies.

Author Contributions: Formal analysis, M.Ś.; methodology, M.Ś.; investigation, M.Ś. and J.K.; supervision, A.K.; writing—original draft preparation, M.S. and A.K.; writing—review and editing, M.Ś., J.K. and A.K. All authors have read and agreed to the published version of the manuscript.

Funding: The publication costs were met through the financial support of the Ministry of Science and Higher Education under the program entitled "Regional lnitiative of Excellence" for the years 2019-2022, Project No. 010/RID/2018/19, amount of funding 12.000.000 PLN.

Institutional Review Board Statement: According to information from the Local Ethics Committee in Olsztyn, no special approval was necessary for the experiments performed under field conditions. All animal procedures, vaccination, and sample collection were performed during standard veterinary inspections and observations.

Data Availability Statement: Data is contained within the article. The datasets used and/or analyzed during the current study are available from the corresponding author on reasonable request.

Acknowledgments: The authors would like to thank Ewa Szczucińska and Edyta Kaczorek-Łukowska from the Department of Microbiology and Clinical Immunology, University of Warmia and Mazury, Olsztyn, Poland, for the help with the microbiological study.

Conflicts of Interest: The authors declare no conflict of interest.

\section{References}

1. Panth, Y. Colibacillosis in poultry: A review. J. Agric. Nat. Resour. 2019, 2, 301-311. [CrossRef]

2. Dho-Moulin, M.; Fairbrother, J.M. Avian pathogenic Escherichia coli (APEC). Vet. Res. 1999, 30, 299-316. [PubMed]

3. Davis, M.; Morishita, T.Y. Relative ammonia concentration, dust concentration and presence of Salmonella species and Echerichia coli inside and outside commercial layer facilities. Avian Dis. 2005, 49, 30-35. [CrossRef]

4. La Ragione, R.M.; Woodward, M.J.; Kumar, M.; Rodenberg, J.; Fan, H.; Wales, A.D.; Karaca, K. Efficacy of a live attenuated Escherichia coli O78:K80 vaccine in chickens and turkeys. Avian Dis. 2013, 57, 273-279. [CrossRef] [PubMed]

5. Rawiwet, V.; Chansiripornchai, N. The efficacy of Escherichia coli aroA-live vaccine in broilers against avian E. coli serotype O78 infection. Thai J. Vet. Med. 2009, 39, 337-342.

6. Śmiałek, M.; Kowalczyk, J.; Koncicki, A. Influence of vaccination of broiler chickens against Escherichia coli with live attenuated vaccine on general properties of E. coli population, IBV vaccination efficiency, and production parameters-A field experiment. Poult. Sci. 2020, 99, 5452-5460. [CrossRef] [PubMed]

7. Galal, H.M.; Tawfek, A.M.; Abdrabou, M.I.; Hessain, A.M.; Alhaaji, A.H.; Kabli, S.A.; Elbehiry, A.; Alwarhi, W.A.; Moussa, I.M. Recent Approaches for Control of E. coli and Respiratory Complex in Middle East. Saudi. J. Biol. Sci. 2018, 25, 1302-1307. [CrossRef] [PubMed] 
8. $\quad$ Davis, G.S.; Waits, K.; Nordstrom, L.; Grande, H.; Weaver, B.; Papp, K.; Horwinski, J.; Koch, B.; Hungate, B.A.; Liu, C.M.; et al. Antibiotic-resistant Escherichia coli from retail poultry meat with different antibiotic use claims. BMC Microbiol. 2018, 18, 174 . [CrossRef] [PubMed]

9. Ibrahim, R.A.; Cryer, T.L.; Lafi, S.Q.; Basha, E.; Good, L.; Tarazi, Y.H. Identification of Escherichia Coli From Broiler Chickens in Jordan, Their Antimicrobial Resistance, Gene Characterization and the Associated Risk Factors. BMC Vet. Res. 2019, 15, 159. [CrossRef] [PubMed]

10. Shrestha, A.; Bajracharya, A.M.; Subedi, H.; Turha, R.S.; Kafle, S.; Sharma, S.; Neupane, S.; Chaudhary, D.K. Multi-drug Resistance and Extended Spectrum Beta Lactamase Producing Gram Negative Bacteria From Chicken Meat in Bharatpur Metropolitan, Nepal. BMC Res. Notes 2017, 10, 574. [CrossRef] [PubMed]

11. Nhung, N.T.; Chansiripornchai, N.; Carrique-Mas, J.J. Antimicrobial Resistance in Bacterial Poultry Pathogens: A Review. Front. Vet. Sci. 2017, 4, 126. [CrossRef] [PubMed]

12. Sweeney, M.T.; Diaz-Campos, D.V.; Bowden, R.; Fritsche, T.R.; Hayes, J.; Langston, C.; Lubbers, B.; Martin-Jimenez, T.; Miller, C.; Pallotta, C.; et al. Performance Standards for Antimicrobial Disk and Dilution Susceptibility Tests for Bacteria Isolated from Animals; VET01; Clinical and Laboratory Standards Institute: Wayne, PA, USA, 2019; pp. 1-156.

13. Kabir, S.M.L. Avian colibacillosis and salmonellosis: A closer look at epidemiology, pathogenesis, diagnosis, control and public health concerns. Int. J. Environ. Res. Public Health 2010, 7, 89-114. [CrossRef] [PubMed]

14. El-Mawgoud, A.I.A.; El-Nahass, E.S.; Shany, S.A.S.; EL-Sawah, A.A.; Dahshan, A.H.M.; Nasef, S.A.; Ali, A. Efficacy of Live Attenuated Vaccine and Commercially Available Lectin Against Avian Pathogenic E. coli Infection in Broiler Chickens. Vet. Sci. 2020, 7, 65. [CrossRef] [PubMed]

15. Fernandes, F.T.; Fávaro, C., Jr.; Ingberman, M.; Beirão, B.C.; Inoue, A.; Gomes, L.; Caron, L.F. Effect of spray Escherichia coli vaccine on the immunity of poultry. Avian Dis. 2013, 57, 671-676.

16. Chapman, H.D.; Jeffers, T.K. Vaccination of chickens against coccidiosis ameliorates drug resistance in commercial poultry production. Int. J. Parasitol. Drugs Drug. Resist. 2014, 4, 214-217. [CrossRef] [PubMed]

17. Chapman, H.D. Sensitivity of field isolates of Eimeria to monensin following the use of a coccidiosis vaccine in broiler chickens. Poult. Sci. 1994, 73, 476-478. [CrossRef] [PubMed]

18. Chapman, H.D.; Jeffers, T.K. Restoration of sensitivity to salinomycin in Eimeria following 5 flocks of broiler chickens reared in floor-pens using drug programs. Poult. Sci. 2015, 94, 943-946. [CrossRef] [PubMed]

19. Śmiałek, M.; Kowalczyk, J.; Gesek, M.; Kaczorek-Łukowska, E.; Dziewulska, D.; Tykałowski, B.; Koncicki, A. The influence of maternally derived antibodies on protection against aMPV/A infection in TRT vaccinated turkeys. Poult. Sci. 2021, 100, 101086. [CrossRef] [PubMed]

20. Śmiałek, M.; Pestka, D.; Tykałowski, B.; Stenzel, T.; Koncicki, A. Development of vaccine-induced immunity against TRT in turkeys depends remarkably on the level of maternal antibodies and the age of birds on the day of vaccination. BMC Vet. Res. 2015, 11, 28. [CrossRef] [PubMed] 